\title{
Desenvolvimento e aplicação de uma tecnologia educacional para auxiliar a comunicação entre o deficiente auditivo e enfermeiro: relato de experiência
}

Development and application of educational technology to assist communication between the hearing impaired and nurse: experience report

Desarrollo y aplicación de la tecnología educativa para ayudar a la comunicación entre el discapacitado auditivo y la enfermera: informe de la experiencia

Waleria do Socorro Rodrigues Oliveira ${ }^{1 *}$, Daniele Melo Sardinha², Ellem Fiel Franco¹, Erivelton do Carmo Baia ${ }^{1}$, João Paulo Duarte Pereira ${ }^{1}$, Rita de Cássia Siqueira Gonçalves ${ }^{1}$, Marcia Andrea da Gama Araújo ${ }^{1}$

\section{RESUMO}

Objetivo: Desenvolver e aplicar uma tecnologia educacional para auxiliar a comunicação entre o deficiente auditivo e enfermeiro. Relato da Experiência: Tratou-se de um estudo descritivo do tipo relato de experiência, em que na primeira etapa, se desenvolveu uma tecnologia educativa, a partir da pesquisa bibliográfica, e na segunda etapa aplicou-se através da Educação Permanente realizada com enfermeiros atuantes do Serviço Público de Saúde. Após a verbalização do tema, da apresentação da tecnologia e de todos terem participado de forma efetiva e correta da dinâmica, abriu-se um espaço para perguntas, possíveis dúvidas e complementação do tema a fim de um retorno avaliativo dos enfermeiros. Sendo a atividade avaliada como positiva pelos enfermeiros da Unidade. Considerações finais: A inclusão da pessoa surda nos Sistemas Públicos de Saúde é um desafio. Ter uma tecnologia que visa a comunicação pelo uso da língua brasileira de sinais (Libras), evidência a importância do profissional de enfermagem em ter o domínio das Libras e, facilitaria a comunicação clara entre o enfermeiro e a pessoa surda em todos os serviços de saúde.

Palavras-chaves: Tecnologia educacional, Comunicação efetiva, Inclusão da pessoa surda, Enfermagem.

\section{ABSTRACT}

Objective: To develop and apply an educational technology to help communication between the hearing impaired and the Nurse. Experience Report: This was a descriptive study of the experience report type, in which in the first stage, an educational technology was developed, based on bibliographic research, and in the second stage it was applied through Permanent Education carried out with Nurses working in the Public Health Service. After the verbalization of the theme, the presentation of the technology and everyone participated effectively and correctly in the dynamics, a space was opened for questions, possible doubts and complementation of the theme in order to give an evaluative return to the Nurses. Being the activity evaluated as positive by the Unit Nurses. Final considerations: The inclusion of deaf people in Public Health Systems is a challenge. Having a technology that aims at communication through the use of the Brazilian sign language (Libras), evidences the importance of the Nursing Professional in having the mastery of Libras and, would facilitate clear communication between the Nurse and the deaf person in all health services.

Key words: Educational technology, Effective communication, Inclusion of the deaf person, Nursing.

\footnotetext{
${ }^{1}$ Centro Universitário Metropolitano da Amazônia (UNIFAMAZ), Belém - PA.

*E-mail: oliveirawaleria29@gmail.com

2 Instituto Evandro Chagas (PPGEVS/IEC). Ananindeua - PA.
} 


\section{RESUMEN}

Objetivo: Desarrollar y aplicar una tecnología educativa para facilitar la comunicación entre el discapacitado auditivo y la enfermera. Informe de la experiencia: Se trata de un estudio descriptivo del tipo de informe de la experiencia, en el que en una primera etapa se desarrolló una tecnología educativa, basada en la investigación bibliográfica, y en una segunda etapa se aplicó a través de la Educación Permanente realizada con las Enfermeras que trabajan en el Servicio de Salud Pública. Después de la verbalización del tema, la presentación de la tecnología y de que todos participaran efectiva y correctamente en la dinámica, se abrió un espacio para preguntas, posibles dudas y complementación del tema para dar un retorno evaluativo a las Enfermeras. Siendo la actividad evaluada como positiva por las Enfermeras de la Unidad. Consideraciones finales: La inclusión de la persona sorda en los sistemas de salud pública es un reto. El hecho de tener una tecnología que apunta a la comunicación a través del uso de la lengua de signos brasileña (Libras), evidencia la importancia del profesional de enfermería en el dominio de la Libras y, facilitaría la comunicación clara entre la enfermera y la persona sorda en todos los servicios de salud.

Palabras clave: Tecnología educativa, Comunicación efectiva, Inclusión de la persona sorda, Enfermería.

\section{INTRODUÇÃO}

Desde o início da humanidade, os indivíduos com algum tipo de limitação física, sensorial ou cognitiva sempre estiveram presentes na sociedade, na antiguidade até os dias atuais as pessoas com deficiência enfrentam problemas e preconceitos com base em estereótipos que dificultam o acesso à saúde. Assim, a adoção do uso da linguagem inclusiva e exclusiva, e de um modelo de saúde que considera o perfil social e demográfico dessa parte da população é o primeiro passo para a melhor inclusão e acessibilidade a saúde na construção e execução de políticas públicas (UNA-SUS, 2017).

A historicidade dos surdos é pouco conhecida na antiguidade, assim havendo relatos e registros de preconceito, discriminação, violência onde estes indivíduos eram abandonados e esquecidos pela sociedade. Entre diversos povos, os surdos eram tidos inábeis de executar e/ou fazer tarefas, em alguns casos eram considerados incapazes de desenvolver a consciência humana. (SILVA EF e CAMPOS MF, 2017). Já na Idade Média, os cristãos acreditavam que diferentemente dos ouvintes, os surdos, uma vez que eram incapazes de proferir os sacramentos não possuíam uma alma imortal, chegando assim a Idade Moderna, onde se distingue pela primeira vez surdez de mudez e a expressão surdo mudo deixa de ser usada para designar o surdo, sendo John Beverley considerado por muitos o primeiro educador dos surdos. (SILVA EF e CAMPOS MF, 2017).

Ao analisar todo o contexto histórico na tentativa de inclusão social de pessoas surdas, observa-se que é primordial a comunicação das mesmas para a interação em sociedade, já que, a estimativa de pessoas surdas é consideravelmente relevante atualmente e, segundo os últimos dados do IBGE em 2013, cerca de 2,2 milhões de pessoas no Brasil possuem deficiência auditiva, onde na região Norte encontra-se a menor proporção verificada $(0,8 \%)$. Os dados coletados foram de pessoas que possuíam surdez bilateral, ou surdez em um ouvido e audição diminuída no outro, ou audição diminuída nos dois ouvidos. (IBGE, 2013). Esse número se torna significativo quando o enfoque é a saúde no Brasil, pois mesmo a língua brasileira de sinais (Libras) sendo considerada a segunda língua do país, muitos profissionais da saúde não possuem domínio sobre ela, dificultando muitas vezes os cuidados de saúde, assim sendo, o uso das Libras na saúde, tem por finalidade melhorar a comunicação efetiva. (GOMES, LF, et al., 2017).

Sabe-se que a comunicação efetiva juntamente com equipe multiprofissional, é entendida como parte importante na segurança do paciente, todavia encontra-se falhas na comunicação dos profissionais de saúde, ocasionando algumas vezes erros irrefutáveis e consequentemente diminuição na qualidade da assistência. (OLINO L, et al., 2019). A segurança do paciente consiste na forma de reduzir riscos de danos dispensáveis ao menor possível, entre as quais a comunicação efetiva faz parte das seis Metas da Organização Mundial da Saúde (OMS) na segurança do paciente, levando em consideração elementos contínuos e profundamente relacionados com comunicação adequada não esquecendo a especificidade de cada um. (SILVA AT, et al., 
2016). Em conformidade com a Lei $n-13.146 / 2015$, Art. 3 do inciso V da Pessoa com Deficiência, garante a inclusão na acessibilidade e conceitua a comunicação como forma de interação dos cidadãos que abrange, entre outras opções, as línguas, inclusive a Língua Brasileira de Sinais (Libras), afirmando o uso de uma linguagem própria, incluindo as tecnologias da informação e das comunicações; (BRASIL, 2015).

Entretanto, as pessoas surdas enfrentam obstáculos descomunais na comunicação, não usufruem de um acesso facilitado dentro dos serviços de saúde, pois não dispõem da linguagem oral e, a maioria dos ouvintes possuem certa dificuldade para compreensão da linguagem dos sinais. (OLIVEIRA YCA, et al., 2015).

O direito de pessoas com deficiência à saúde, intercalando com os direitos humanos, a fim de um Plano Nacional de Direito da Pessoa com Deficiência, com direito aos bens e serviços de saúde sem discriminação dando origem a uma Rede de Cuidado à Pessoa com Deficiência, entre os serviços está a qualificação das equipes de saúde da atenção básica. (UNA-SUS, 2017). Para garantir o direito e o acesso nos Serviços Públicos de Saúde, a Lei no 13.146/2015 no Art. 18, assegura à Pessoa com Deficiência, atenção integral em todos os níveis de complexidade, por intermédio do SUS, garantindo o acesso universal e igualitário. (BRASIL, 2015).

Desse modo, criou-se uma tecnologia para desenvolver um Relato de Experiência, fazendo parte das atividades curriculares acadêmicas. Refletindo sobre o tema abordado, visando a equidade que é uma das diretrizes do Sistema Único de Saúde (SUS), com finalidade de melhorar a inclusão e comunicação entre o profissional enfermeiro junto aos usuários que necessitam dessa atenção diferencia, este trabalho teve como objetivo desenvolver e aplicar uma tecnologia educacional para auxiliar a comunicação entre o deficiente auditivo e enfermeiro.

\section{RELATO DA EXPERIÊNCIA}

O trabalho tratou-se de um estudo descritivo do tipo relato de experiência, que procurou agregar por meio da Educação Permanente, junto aos enfermeiros atuantes do Serviço Público de Saúde, através do desenvolvimento de uma tecnologia inclusiva das pessoas surdas dentro do SUS, na busca da melhor Assistência de Enfermagem pelo uso adequado da comunicação efetiva.

O processo de criação da tecnologia foi a primeira etapa deste estudo, através de bases on-line representantes da língua dos sinais Libras para comunicação, a partir de então, criou-se um personagem denominado como "Guguinha". O processo da criação do personagem dividiu-se em duas etapas, a primeira fez-se em papel A4 e, posteriormente passado para mídia digital, finalizando a tecnologia em um painel. No painel, o personagem associava-se a tecnologia demonstrando gestos em Libras que visava simplificar o entendimento do enfermeiro quanto a queixas de sinais e sintomas básicos. Sendo os sinais: de alergia, ansiedade, dor, enjoo, insônia, medo, tontura e tosse. Associado ou não a um período de tempo, tais como: 1 dia, 1 semana e 3 semanas. O painel ilustrativo continha informações para o uso adequado das Libras, ao primeiro contato do enfermeiro com pessoa surda.

O estudo bibliográfico foi baseado em literaturas obtidas através de pesquisas em artigos científicos oriundos de plataformas on-line (A Scientific Electronic Library Online - Scielo, Biblioteca Virtual em Saúde BVS, Lei № 13.146/2015 - L13146 Planalto, Universidade Aberta do SUS- UNA-SUS, Pesquisa Nacional de Saúde - PNS e Instituto Brasileiro de Geografia e Estatística- IBGE), no período de 2013 a 2019. Usaram-se com descritores Tecnologia Educacional, Comunicação Efetiva, Inclusão da Pessoa Surda, Enfermagem. O estudo bibliográfico inicial sobre comunicação efetiva, inspiraram formas já presentes do uso das Libras, a fim de adaptá-las à realidade dos serviços públicos de saúde na assistência de Enfermagem na segurança do paciente.

Dentre vários tipos de Serviços Públicos de Saúde existentes, escolheu-se para trabalhar a pessoa surda por meio da tecnologia inclusiva, enfermeiros da Unidade Básica de Saúde (UBS), pois, a Atenção Básica (AB) é a porta de entrada para os serviços do SUS. Sendo assim, este projeto tem por intuito alcançar todo profissional enfermeiro em qualquer âmbito de prestação da saúde pública, alcançando até mesmo o âmbito hospitalar, objetivando a segurança do paciente surdo. A unidade em questão trata-se de uma Estratégia

REAS/EJCH | Vol.Sup.n.44 | e2269 | DOI: https://doi.org/10.25248/reas.e2269.2020 Página 3 de 6 
Saúde da Família (ESF), a mesma, contém duas equipes, cada equipe tem um médico, um enfermeiro, um técnico de Enfermagem e três agentes comunitários de saúde (ACS). A unidade contém Farmácia, recepção, triagem, sala de vacina, consultório médico, consultório de enfermagem, sala de reunião, sala de esterilização e sala de curativo.

A aplicação da tecnologia foi realizada em 6 passos, onde o primeiro passo deu-se pela apresentação de cada membro da equipe dos acadêmicos de Enfermagem. O segundo passo, foi uma breve introdução sobre a historicidade dos surdos e sua dificuldade de acesso nas unidades de saúde, elaborou-se um cartaz com nuvens de ideias, apresentadas por meio de um fluxograma sobre o referido tema. O terceiro passo, deu-se a partir da apresentação da tecnologia em banner, que continham imagens dos sinais em Libras a respeito das principais queixas e tempo do ocorrido, para os usuários surdos do sistema de saúde, houve também, a demonstração em Libras do que continham no painel. No quarto passo, simulou-se uma situação a partir da chegada de um paciente surdo dentro do serviço, a fim de uma consulta de Enfermagem, onde o enfermeiro se depararia pela primeira vez com a dificuldade em comunicar-se com este paciente e, a tecnologia o auxiliou na utilização dos sinais em Libras do painel. No quinto passo, aconteceu uma dinâmica com os profissionais enfermeiros para fixação dos sinais, os mesmos precisavam associar figuras que continham os sinais em Libras a um segundo painel que continham apenas as queixas escritas. O sexto passo, foi aberto uma roda de conversa para perguntas e possíveis dúvidas que os profissionais poderiam apresentar, falar da importância do tema e, da criação de uma tecnologia para as pessoas surdas.

A ação iniciou-se às $15 \mathrm{~h}$ e $20 \mathrm{~min}$, com término às 17:00h e, estavam presentes na Unidade durante a Educação Permanente dois enfermeiros, duas acadêmicas de Enfermagem, duas Técnicas de Enfermagem e cinco Agentes Comunitários de Saúde (ASC). Fez-se primeiramente a verbalização do tema, para o entendimento do que seria discutido e apresentação da tecnologia. Após a verbalização do tema, da apresentação da tecnologia e de todos terem participado de forma efetiva e correta da dinâmica, abriu-se um espaço para perguntas, possíveis dúvidas e complementação do tema a fim de um retorno avaliativo dos enfermeiros, quanto ao desenvolvimento da tecnologia e da didática utilizada, sobre o quanto estas poderiam auxiliar na melhora, na qualidade e atendimento às pessoas surdas usuárias dos Sistemas Público de Saúde.

A enfermeira relatou que atua como servidora na Secretaria Saúde do Município há um ano e, a ação foi de suma importância, pois não se sente preparada para atender pacientes surdos, salienta ainda, que toda a equipe de saúde precisa receber uma capacitação deste tipo, constantemente. Enfatizando ainda, que a tecnologia é dinâmica e específica para as sintomatologias básicas em indivíduos que chegam nos serviços de saúde, e a fixação da tecnologia nos serviços públicos de saúde facilita a comunicação, tendo por objetivo de que comunidade saiba que há profissionais qualificados em atendê-los, finalizou e classificou a ação como positiva. O enfermeiro, em sua fala, afirmou o quão é necessário o uso das Libras, pois narrou o fato de existir em sua família uma criança surda. Admitindo que precisa entender e saber usar a língua dos sinais para uma melhor comunicação, já que, não possui domínio sobre a língua brasileira de sinais (Libras).

\section{DISCUSSÃO}

A experiência como acadêmicos de Enfermagem, frente às pesquisas e a apresentação da tecnologia, pôde agregar conhecimentos para a nossa melhor formação e atuação como futuros enfermeiros frente às diversidades. Observou-se entre os profissionais de Enfermagem, que a implantação de um novo método por meio de uma tecnologia inclusiva, para com os deficientes auditivos, ainda não se fazia presente. $O$ uso das tecnologias educativas possibilita o processo de ensino e aprendizagem. A avaliação dos discentes colocaos como coautores, a fim da ruptura do estigma da aprendizagem tradicional. (GADELHA, MMT, et al., 2019).

A partir da pesquisa, foi observado que são raras as formas de uma tecnologia para pessoas surdas nos Sistemas de Saúde. Desta forma, a ideia de utilizar um banner com figuras descritivas como gamificação na Educação Permanente, teve por finalidade levar aos profissionais enfermeiros novos instrumentos para uma comunicação adequada, frente a situações reais associadas a pessoas com deficiência auditiva. A Educação Permanente é uma ferramenta essencial para capacitar e realizar mudança no ambiente de trabalho, para que os profissionais consigam solucionar quaisquer dificuldades durante a assistência, que irão influenciar a

REAS/EJCH | Vol.Sup.n.44 | e2269 | DOI: https://doi.org/10.25248/reas.e2269.2020 Página 4 de 6 
organização do trabalho, a fim de que os trabalhadores tenham habilidades de suprir as necessidades individuais e coletivas nos serviços de saúde. Que consequentemente gerará processos de crescimento da instituição. (FRANÇA NGGM e SILVA RG, 2018).

Um estudo realizado no Hospital Israelita Albert Einstein (HIAE), com 37 enfermeiros e 60 técnicos de enfermagem, avaliou quais estratégias eles utilizavam para se comunicar com pacientes surdos, e demonstrou que 100\% utilizavam mímica, leitura labial 92\% enfermeiros e 95\% técnicos, Libras 0\% enfermeiros e $2 \%$ técnicos, escrita $19 \%$ enfermeiros e $56 \%$ técnicos, auxílio do acompanhante $43 \%$ enfermeiros e $78 \%$ técnicos, outros $0 \%$ enfermeiros e $3 \%$ técnicos BRITTO FR e SAMPERIZ MMF, 2010).

Nesse contexto, a utilização de instrumentos para facilitar a comunicação entre profissional da saúde e indivíduo surdo, sendo verbal ou não verbal, deve estar inserida no processo terapêutico, pois, irá repercutir na qualidade do atendimento, garantia do direito à saúde ao indivíduo, como também uma forma de acolhimento, e promoção da saúde da população surda. Nesse contexto, a elaboração de tecnologias é primordial para mediar essa relação, ocasionado em diversos benefícios tanto para o profissional e paciente (RAMOS TS e ALMEIDA MAPT, 2017).

Ao princípio de equidade, diz atender o indivíduo de acordo com sua necessidade e, uma das formas em atendê-los seria, construir a ideia do quão importante é a disciplina Libras na grade curricular de qualquer curso de graduação, especialmente na área da saúde, já que esta é uma disciplina optativa para áreas que não seja o magistério ou fonoaudiologia. O Estado criou a Lei no 10.436/2002 e o decreto no 5.626/2005 que estabelece no Art. $3^{\circ}$ como disciplina obrigatória para os cursos descritos e no $\S 2^{\circ}$ como disciplina optativa em outras áreas da graduação (OLIVEIRA YCA, et al., 2012).

Frente a discussão, refletiu-se de que há a necessidade da inclusão das Libras como disciplina obrigatória na grade curricular dos cursos de Graduação em Enfermagem. Na execução do tema abordado, evidenciouse que há uma carência para atender o deficiente auditivo e, que pouco é falado sobre a qualificação dos enfermeiros voltado para melhorias no atendimento desses usuários. A inserção do curso de Libras de forma integral na graduação, permite ao profissional de qualquer área a comunicação efetiva, permitindo-Ihe ser um diferencial em sua área de atuação, consequentemente reparando possíveis falhas no SUS. O curso de Libras possibilita a equidade de cada indivíduo e, sustenta a ideia dos direitos fundamentais da pessoa com deficiência, supriria o déficit na falha da assistência diferenciada ao paciente deficiente auditivo, já que, os profissionais mostram-se não estar preparados para atender de forma específica a esse público. (FRANÇA, NGGM e SILVA RG, 2018).

\section{CONSIDERAÇÕES FINAIS}

Trabalhar a inclusão da pessoa surda nos Sistemas Públicos de Saúde é um desafio, e há uma necessidade de pesquisas sobre o tema, no que se refere a inclusão do paciente. Pois, uma tecnologia que visa a comunicação pelo uso da língua brasileira de sinais (Libras), evidência a importância do Profissional de Enfermagem em utilizá-la, uma vez que este profissional ao mostrar-se capaz de comunicar-se com a pessoa surda por meio das Libras sobre as sintomatologias básicas expostas na tecnologia apresentada, só fortalece a ideia de que comunicação efetiva é uma ferramenta essencial para a segurança do paciente, em especial os pacientes surdos, a fim de uma manter uma excelente Assistência de Enfermagem em todos os aspectos e limitações existentes do ser humano.

\section{REFERÊNCIAS}

1. BRASIL, Instituto Brasileiro de Geografia e Estatística (IBGE) censo 2010. Pesquisa Nacional de Saúde (PNS) 2013.

2. BRASIL, Lei № 13.146, DE 6 DE JULHO DE 2015.

3. BRITTO FR, SAMPERIZ, MMF. Dificuldades de comunicação e estratégias utilizadas pelos enfermeiros e sua equipe na assistência ao deficiente auditivo. Einstein (São Paulo), 2010, 8(1): 80-85.

4. FRANÇA NGGM, SILVA RG. Percepção de Enfermeiros Sobre a Comunicação no Atendimento à Pessoa com Deficiência Auditiva. Revista Brasileira de Ciências da Vida, 2018. 
5. GADELHA MMT, et al., 2019. Tecnologias Educativas no Processo Formativo: Discurso dos Acadêmicos de Enfermagem. Revista de Enfermagem UFPE on line, 2019, 13(1): 155-161.

6. GOMES LF, et al. Conhecimento de Libras pelos Médicos do Distrito Federal e Atendimento ao Paciente Surdo. Revista Brasileira de Educação Médica, 2017, 41(3); 390-396.

7. OLINO L, et al. Comunicação efetiva para a segurança do paciente: nota de transferência e Modified Early Warning Score. Revista Gaúcha de Enfermagem, 2019, 40(esp); e20180341.

8. OLIVEIRA AEF, GARCIA PT. Redes de Atenção à Saúde: Rede de Cuidados à pessoa com Deficiência. São Luis: MA, 2017; 15p e 51p.

9. OLIVEIRA YCA, et al. Comunicação como Ferramenta Essencial para Assistência à Saúde dos Surdos. Physis [online], 2015, 25(1); 307-320.

10. RAMOS TS, ALMEIDA MAPT. A importância do ensino de libras: relevância para profissionais de saúde. Id on line Revista Multidisciplinar e de Psicologia, 2017; 10(33): 116-126.

11. SILVA AT, et al. Assistência de enfermagem e o enfoque da segurança do paciente no cenário brasileiro. Saúde debate [online]. 2016, vol.40, n.111, pp.292-301.

12. SILVA EF, CAMPOS MF. O Percurso dos Surdos na História e a Necessidade da Libras para a Inclusão dos Sujeitos na Escola. JOIN, Editora Realize, 2017. 